\title{
Design and validation of a scoring system for rapid identification of severe acute pancreatitis in patients with acute pancreatitis
}

\section{Ling Wang ( $\sim 463082910 @ q q . c o m$ )}

PEOPLIS HOSPITAL OF QIANDONGNAN MIAO AND DONG https://orcid.org/0000-0001-7114-0519

\section{Shaohong Wang}

Peking University People's Hospital

Jingguo Zhang

the first people's hospitial of KAILI

\section{Research}

Keywords: acute pancreatitis, severe acute pancreatitis, critical illness, rapid identification, scoring

Posted Date: January 23rd, 2020

DOI: https://doi.org/10.21203/rs.2.21711/v1

License: (c) (i) This work is licensed under a Creative Commons Attribution 4.0 International License.

Read Full License 


\section{Abstract}

Purpose: Early identification of SAP and take necessary treatment can reduce the mortality rate of patients with AP. This study aimed to design a scoring system for rapid identification of SAP (RISAP) and evaluate its performance in predicting SAP in patients with AP.

Methods: In the first phase, 1024 patients with AP who were admitted to the people's hospital of Qiandongnan Miao and Dong Autonomous Prefecture ,The Second Affiliated Hospital of Guizhou Medical University,the First People's Hospital of Kaili from January 2015 to June 2017 were included. Easily obtained indicators including patients' gender, age, previous history of pancreatitis, acute diffuse peritonitis (ADP), pleural effusion (PE), heart rate (HR), respiratory rate (RR), and systolic blood pressure (SBP) measured under adequate analgesia, quietness conditions at admission were selected. Logistic regression analysis was performed to identify the risk factors for SAP. After determination of the cutoff values of the identified risk factors using ROC curve analysis, RISAP scoring system was designed. In the second phase, a total of 740 patients with AP who were admitted to our hospital from July 2017 to October 2019 were included and divided into SAP and non-SAP groups. RISAP, RANSON and BISAP scores were measured and compared between groups. The ROC curve was draw to analyze the ability of RISAP score in predicting SAP.

Results: The number of patients who had history of pancreatitis,ADP, PE, HR, BR were significantly higher in the SAP group than in the non-SAP group $(P<0.05)$. Logistic regression analysis showed that $P E, H R$, and RR were independent risk factors for SAP. Then RISAP score was designed based on the cutoff values of the three risk factors $(0.5,95.5,22.5$, respectively). The RISA, RANSON, and BISAP scores were significantly higher in the SAP group than that in the non-SAP group $(U=-9.501,-3.701,-8.520 P<0.05)$. Compared with the RANSON, and BISAP scores, RISAP had the highest AUC values, sensitivity and specificity.

Conclusion: The designed RISAP score is simple, convenient, economical, non-invasive, and highly repeatable, which is superior in rapid identification of SAP in patients with AP.

\section{Introduction}

Acute pancreatitis (AP) is a one of the most common diseases of the gastrointestinal tract, which can develop into severe acute pancreatitis (SAP) that rapidly progresses from a local tissue damage into multiple organ dysfunction. The incidence rate of SAP is about $20 \%-40 \%$ [1]. SAP is a dangerous disease with rapid progression, several complications, and a high mortality rate of up to $30 \%-50 \%[2,3]$. Early identification of SAP is critical to reduce the mortality rates in patients with AP [4].

At present, a number of scoring systems have been developed to estimate the severity of AP, such as Ranson's score, bedside index for severity in acute pancreatitis (BISAP) score, acute physiology and

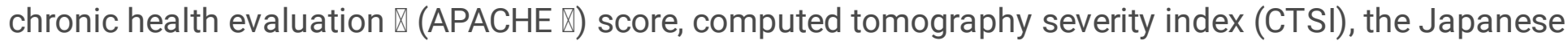
Severity Score (JSS). However, those scoring systems have disadvantages, which are difficult to 
memorize, invasive, complex, not reproducible, and have a time delay [5], so their use is limited. The purpose of our study was to design and develop a scoring system for rapid identification of severe acute pancreatitis (RISAP) in patients with acute pancreatitis and verify the predictive value of RISAP score in identifying SAP in AP patients.

\section{Subjects And Methods}

The study was conducted in two phases. In the first phase, we designed the RISAP score, and in the second phase, we verified the ability of RISAP score in predicting SAP in patients with AP.

\section{Design of the RISAP score}

A total of 1024 patients with AP who were admitted to the people's hospital of Qiandongnan Miao and Dong Autonomous Prefecture,The Second Affiliated Hospital of Guizhou Medical University,the First People's Hospital of Kaili from January 2015 to June 2017 were included. There were 552 males and 472 females with an average age of $50.10 \pm 14.35$ years (range, 18-80 years). Among these 1024 patients, 280 patients had SAP, 744 patients had AP, and 232 patients had a history of pancreatitis.

Inclusion criteria were: 1) aged 18-80 years; 2) patients met the diagnostic criteria for Acute pancreatitis according to the revised Atlanta classification (RAC) 2012 [6].

Exclusion criteria were: patients with malignant tumors, immunodeficiency, cirrhosis, uremia, abdominal trauma, and pleural effusion (PE) that is not caused by AP; critically ill patient with cardiac and respiratory diseases; patients who refused to receive treatment.

Treatment methods included gastrointestinal decompression, inhibition of pancreatic enzyme secretion and activities, analgesia, nutritional support and symptomatic treatment. Antibiotics should be used when infection occurs, abdominal drainage, mechanical ventilation and bedside continuous renal replacement therapy (CRRT) were given if necessary. Patients with biliogenic severe pancreatitis (SP) and cholangitis underwent endoscopic retrograde cholangiopancreatography (ERCP) and endoscopic sphincterotomy within 24 hours after admission.

\section{RISAP design method}

The selection of indicators: it will take a long time to obtain laboratory test results of patients, so laboratory indicators, such as blood urea nitrogen, white blood cells, calcium ions, partial oxygen pressure, hematocrit, blood glucose, lactate dehydrogenase were not selected. Indicators that were easily obtained were selected, including patients' gender, age, previous history of pancreatitis, acute diffuse peritonitis (ADP), pleural effusion (PE), heart rate (HR), respiratory rate (RR), and systolic blood pressure (SBP) measured under optimal conditions (adequate analgesia, quietness,Sedation-Agitation Scale score was 4 points) at admission. (2) Grouping: Patients were divided into SAP and non-SAP groups based on whether the patients had developed SAP within 7 days of admission. (3) Determination of the cut-off value of the risk factors for SAP: differences in gender, age, previous history of pancreatitis, ADP, PE, HR, 
RR, and SBP between the two groups were analyzed. Logistic regression analysis was performed to identify the independent risk factors for SAP. Receiver operating characteristic (ROC) curve analysis was conducted to evaluate the ability of independent risk factors in predicting SAP and determine the cut-off values. (4) Design of RISAP score: RISAP was designed according to the cut-off values of the identified risk factors.

\section{Validation of the RISAP in patients with AP}

A total of 740 patients with AP who were admitted to the people's hospital of Qiandongnan Miao and Dong Autonomous Prefecture,The Second Affiliated Hospital of Guizhou Medical University,the First People's Hospital of Kaili from July 2017 to October 2019 were included. There were 448 males and 292 females with an average age of $47.66 \pm 15.47$ years (range, $18-80$ years). Among these 740 patients, 304 patients had SAP, 436 patients had AP. Inclusion and exclusion criteria and treatment methods were the same as described above.

All patients were divided into SAP and non-SAP groups based on whether the patients had developed SAP within 7 days of admission. According to PE, and the HR, RR, measured under adequate analgesia , quietness conditions at admission, RISAP score for patients were measured. RANSON and BISAP scores were also measured. The differences in the RISAP, RANSON, and BISAP scores were compared between the two groups, and the ROC curve was draw to analyze the ability of RISAP score in predicting SAP.

\section{Statistical Analysis}

Statistical analysis was performed using SPSS 22.0 software. The Kolmogorov-Smirnov test was used to analyze the normality of the distributions of the measurement data. Measurement data with normal distribution are expressed as mean \pm standard deviation (mean $\pm S D$ ), and comparisons among the groups were done by the one-way ANOVA followed by the LSD test (homogeneity of variance was determined) or the Tamhane's T2 test (homogeneity of variance was not determined). Non-normal distribution data were expressed as median and inter-quartile range [M (QU-QL)], and comparisons among the groups were done by the Mann-Whitney $U$ test. The numeration data were analyzed by chisquare $\left(X^{2}\right)$ test. Logistic regression analysis was performed to identify the risk factors, and ROC curve analysis was conducted to evaluate the ability of RISAP score in predicting SAP. A value of $P<0.05$ was considered statistically significant.

\section{Results}

\section{RISAP Design}

There was no statistically significant difference between SAP and non-SAP groups with respect to age, history of pancreatitis and SBP $(P>0.05)$. Compared with non-SAP group, SAP group had more males than females, HR, BR and the number of patients who had ADP or PE, were significantly higher in the SAP group than in the non-SAP group $(P<0.05$, Table 1$)$. 
Logistic regression analysis showed that $P E, H R$, and RR were independent risk factors for SAP (Table 2). In ROC analysis, the areas under the ROC curve (AUCs) for PE, HB, and BR were $0.764(0.662,0.866)$, $0.932(0.875,0.989)$, and $0.921(0.855,0.986)$, respectively, the odds ratios (OR) were $12.933,1.594$, and 1.151 , respectively. Cutoff values were $0.5,95.5,22.5$, respectively (Table 3 ).

The RISAP designed according to the cutoff values of PE, HR, and BR was shown in Table 4. In order to calculate the score and remember easily, only 0 and 1 point were assigned for each parameter, i.e. 0 point= no $P E ; H R<96 ; R R<23 ; 1$ point $=P E ; H R \geq 96 ; B R \geq 23$. The total RISAP score is the sum of those three scores with 3 being the highest score and 0 being the lowest score.

\section{RISAP validation}

As shown in Table 5, there was no significant difference in gender and age between the SAP and non-SAP groups $\left(\chi^{2} / t=2.347,0.605 P \otimes 0.05\right)$. The RISA, RANSON, and BISAP scores were significantly higher in the SAP group than that in the non-SAP group $(U=-9.501,-3.701,-8.520 \mathrm{P}<0.05)$.

The AUCs for RISAP, RANSON, and BISAP were $0.892(0.842,0.941), 0.657(0.576,0.739)$, and 0.853 $(0.796,0.909)$, respectively; the cutoff values were $1.5,2.5$, and 1.5 , respectively. The sensitivity of RISAP, RANSON, and BISAP in predicting SAP were $79.70 \%, 53.90 \%$, and $75.00 \%$, respectively, the specificity were $96.30 \%, 68.80 \%, 86.40 \%$. Compared with the RANSON, and BISAP scores, RISAP had the highest AUC values, sensitivity and specificity (Figure 1, Table 6).

\section{Discussion}

The Revised Atlanta Classification 2012 defined three degrees of severity of AP: mild AP (MAP), moderate severe AP (MSAP), and SAP. SAP is characterized by persistent organ failure (greater than 48 hours). Patients with AP complicated with multiple organ dysfunction syndromes (MODS) are considered to have SAP. SAP is an extremely dangerous condition, and the in-hospital mortality rate of SAP in China is $11.8 \%$ [7]. Early identification of SAP is necessary to reduce the mortality rate of patients with AP [8].

Currently, there are a number of scoring systems and indicators for assessing the severity of AP. Ranson's score is one of the most widely used clinical prognostic scores. A Ranson's score of $\geq 3$ have a better sensitivity for the diagnosis of SAP [9]. However, Ranson's score can not be used for all populations. YANG et al. showed that Ranson's score has no significant advantage in predicting the severity and prognosis of hyperlipidemic acute pancreatitis (HLAP) [10], and Ranson's score can only be determined after 48 hours of admission, the 48-hour delay in predicting the disease condition may lead to delayed fluid resuscitation and delayed interventions for treatment of complications, and rapid identification of SAP cannot be achieved. APACHEX score is the most widely accepted and used scoring system, and is also a highly recommended scoring system by many guidelines [11]. SAP can be diagnosed when patients had an APACHEX score of $>8$, and those patients need to be transferred to ICU [12]. Study had shown that APACHE II score is a reliable scoring system, and has high sensitivity for assessing the severity, organ failure and death in AP [13]. However, the APACHEX score can only be determined after 24 
hours of admission, and it is a complex system which includes many indicators, and highly depends on laboratory test results, so dynamic determination of disease severity is difficult. BISAP is one of the most accurate and applicable scoring systems in predicting severity, death, and organ failure of patients with AP [14]. BISAP score $\geq 3$ is considered SAP, the specificity of BISAP score in predicting SAP is comparable to RANSON's score [15], and the ability of BISAP score in predicting severity, infectious pancreatic necrosis and mortality rates in AP are comparable to the APACHE II score [16], and BISAP score is easier to calculate. However, this scoring system mainly depends on laboratory indicators, which can not achieve a rapid assessment of SAP. According to the Balthazar's CT score, AP was classified into five grades (A to E), which can evaluate the organ failure in patients with AP [17]. CTSI and the modified CT severity index (MCTSI) include the degree of pancreatic necrosis based on the Balthazar score, which are widely used imaging scoring systems. Enhanced CT score (CECT) has a higher sensitivity in assessing the severity of the disease [18]. However, in order to dynamic monitoring, patients have to undergo numerous CT scans and pay high examination costs. And some patients cannot take dynamic CT examinations due to their inability to move themselves. It has been reported that the CT evidence of SAP lags behind clinical outcomes [19]. One certain shortcoming of CTSI, MCTSI, and CECT is the low reproducibility. JSS includes 9 risk factors and enhanced CT examination scores with relatively high accuracy, which is considered to be the best scoring system for the prediction of organ failure in patients with AP [20], but JSS completely depends on the results of laboratory tests and physical examinations, the feasibility of dynamic assessment of SAP by JSS is relatively low. The modified early warning score (MEWS) can independently predict the poor prognosis and mortality rates of AP patients [21]. MEWS score has the advantages of convenience and non-invasiveness, which can be calculated timely, but MEWS is based on seven indicators, these scores are relatively complex, it is difficult for clinicians to remember with poor compliance. Sequential organ failure (SOFA) is a scoring system used to assess organ function in patients with AP, which can effectively predict severity and mortality of AP[22], but SOFA score requires multiple laboratory tests, which can increase the suffering and economic burden in patients. And it is difficult to remember and complex to score, this limits the compliance of the clinicians. Although the sensitivity and specificity of the above scoring systems or indicators have been partially confirmed, but most of them are more complex to use and do not provide an early assessment of AP [5]. At present, laboratory indicators such as C-reactive protein, blood urea nitrogen, and hematocrit have been used for predicting SAP [1], and have certain predictive value, but too many factors can influence the levels of the above laboratory indicators, laboratory tests are expensive and those indicators are difficult to obtain, so those laboratory indicators may not predict SAP accurately. Some biomarkers such as regulator of calcineurin1, Pentraxin-3, cyclophilin A, microRNA (miRNA), soluble $\mathrm{CD}_{73}$, oleic acid chlorohydrin both have the potential to predict SAP, but those biomarkers have not been used in the clinical practice [5]. As mentioned above, the scoring systems and laboratory indicators currently used to predict SAP both have disadvantages, which may limit their clinical use [23]. Developing a simple, convenient, highly reproducible tool for rapid identification of SAP is needed.

In the present study, we used the simplest and easy-to-obtain indicators to design a rapid identification scoring system for SAP. Logistic regression analysis showed that the history of PE, HR, and RR were 
independent risk factors for SAP with cutoff values of $0.5,95.5,22.5$, respectively. The RISAP score was designed according to the cutoff values of the three risk factors, 0 and 1 point were assigned for each indicator, the highest score is 3 and the lowest score is 0 . HR and RR of patients were measured under adequate analgesia, quietness conditions at admission, pleural effusion can be detected by Doppler ultrasound, CT, bedside evaluation or physical examination. The new designed RISAP score has the ability to predict disease severity timely, it is also simple to use and easy to remember. And in the present study, we used the RISAP score to predict SAP in patients with AP, we found that the RISAP score was significantly higher in the SAP group than that in the non-SAP group, indicating that RISAP can reflect the severity of the AP. When RISAP score $\geq 2$, the AUC, sensitivity and specificity of RISAP in predicting SAP is $0.892(0.842,0.941), 79.70 \%$, and $96.30 \%$, respectively. Compared with RANSON and BISAP scores, RISAP has the highest AUC, sensitivity and specificity. The results indicated that RISAP score is superior in the prediction of SAP.

If patients present with deep sighing respiration or respiratory arrest, it is obvious that the patients are in critical condition, and emergency treatment for sudden cardiac arrest should be adopted, so RISAP score is not suitable to use in those patients. If HR is greater than 180 beats $/ \mathrm{min}$ or less than 40 beats $/ \mathrm{min}$, those patients were considered to have critical cardiac illness, who were not considered to develop SAP. RISAP is also not suitable to use in patients presenting with tuberculous pleurisy, heart failure, and pleural bleeding, and PE that is not caused by AP. And RISAP score may not be appropriate for patients with pancreatic trauma, pancreatic tumors, cirrhosis, uremia or immunodeficiency, which is also not suitable for infants and children.

The limitation of the present study is that during the first design phase, only 280 patients with SAP from three center of Qiandongnan Miao and Dong Autonomous Prefecture were included, this may introduce bias in the results in terms of cutoff values of identified risk factor, For other regions and other races, the practicability of this scoring system needs further research.

\section{Conclusions}

The designed RISAP is a simpler scoring tool that can quickly identify SAP among AP patients. It is convenient, economical, non-invasive, and highly repeatable,Compared with RANSON and BISAP scoring system has some advantages. which is worthy of clinical promotion.

\section{Declarations}

\section{Ethical Approval and Consent to participate}

This study was approved by the ethics committee of the people's hospital of Qiandongnan Miao and Dong Autonomous Prefecture. All patients signed informed consent before inclusion.

\section{Consent for publication}


All the authors agree to publish the paper

\section{Availability of supporting data}

All relevant data are within the paper and the Supporting Information files.

\section{Conflicts of interest}

All the authors declare that they have no conficts of interest.

\section{Funding}

The study was funded by the researchers themselves, with no other funding

\section{Authors' contributions}

Ling Wang's contributions was expert consultation, thesis design, quality control, data search, data collection, statistical analysis and thesis writing

Shaohong Wang's contributions was quality control and data collection

Jingguo Zhang's contributions was quality control and data collection

\section{Acknowledgements}

The authors are grateful to Professor Xianqing Shi ,Professor Feng Shen,Professor Xiaoyun Fu, who helped with the topic development and manuscript improvements.

\section{Authors' information}

Wang ling: major in critical care medicine, associate chief physician, mainly engaged in the study of severe pancreatitis and ARDS.

Wang shaohong: major in critical care medicine, chief physician, mainly engaged in the study of severe acute pancreatitis and severe infection.

Zhang jingguo: major in critical care medicine, attending physician, mainly engaged in the study of severe acute pancreatitis and MODS.

\section{References}


1. Leppäniemi A, Tolonen M, Tarasconi A, Segovia-Lohse H, Gamberini E, Kirkpatrick AW, Ball CG, Parry N, Sartelli M, Wolbrink D, van Goor H, Baiocchi G, Ansaloni L, Biffl W, Coccolini F, Di Saverio S, Kluger Y, Moore E, Catena F (2019) 2019 WSES guidelines for the management of severe acute pancreatitis. World J Emerg Surg 14:27. doi: 10.1186/s13017-019-0247-0.

2. Sileikis A, Beiša V, Beiša A, Samuilis A, Serpytis M, Strupas K (2013) Minimally invasive retroperitoneal necrosectomy in management of acute necrotizing pancreatitis. Wideochir Inne Tech Maloinwazyjne 8:29-35. doi: 10.5114/wiitm.2011.30943.

3. Sandler RS, Everhart JE, Donowitz M, Adams E, Cronin K, Goodman C, Gemmen E, Shah S, Avdic A, Rubin R (2002) The burden of selected digestive diseases in the United States. Gastroenterology 122:1500-1511.doi:10.1053/gast.2002.32978.

4. Ma M X,Jing C,Ju J (2019) Research progress of scoring systems of acute pancreatitis.Chin J Gastroenter Hepatol 28:350-355. doi: 10.3969/j.issn.1006-5709.2019.03.024.

5. Hao J, Chen J (2019) Research advances in prediction of acute pancreatitis severity by biomarkers. Medical Recapitulate 25:1202-1207. do:10.3969/j.issn.1006-2084.2019.06.030.

6. Banks PA, Bollen TL, Dervenis C, Gooszen HG, Johnson CD, Sarr MG, Tsiotos GG, Vege SS; Acute Pancreatitis Classification Working Group (2013) Classification of acute pancreatitis-2012: revision of the Atlanta classification and definitions by international consensus. Gut 62:102-111. doi: 10.1136/gutjnl-2012-302779.

7. Bai Y, Liu Y, Jia L, Jiang H, Ji M, Lv N, Huang K, Zou X, Li Y, Tang C, Guo X, Peng X, Fang D, Wang B, Yang B, Wang L, Li Z (2007) Severe acute pancreatitis in China: etiology and mortality in 1976 patients. Pancreas 35:232-237. doi: 10.1097/MPA.0b013e3180654d20.

8. Forsmark CE, Vege SS, Wilcox CM (2016) Acute Pancreatitis. N Engl J Med 375:19721981.doi:10.1056/NEJMra1505202.

9. Shabbir S, Jamal S, Khaliq T, Khan ZM (2015) Comparison of BISAP Score with Ranson's Score in Determining the Severity of Acute Pancreatitis. J Coll Physicians Surg Pak 25:328-331. doi: 05.2015/JCPSP.328331.

10. Yang L, Liu J, Xing Y, Du L, Chen J, Liu X, Hao J ( 2016) Comparison of BISAP, Ranson, MCTSI, and APACHE II in Predicting Severity and Prognoses of Hyperlipidemic Acute Pancreatitis in Chinese Patients. Gastroenterol Res Pract 2016:1834256. doi: 1155/2016 /1834256区

11. Fang $L$ ( 2013) Recommended guidelines for the management of acute pancreatitis by the American College of Gastroenterology in 2013. Journal of Clinical Hepatology 29:45-51.doi: 10.3969/j.issn.1001-5256.2013.12.002.

12. Harshit Kumar A, Singh Griwan M (2018) A comparison of APACHE II, BISAP, Ranson's score and modified CTSI in predicting the severity of acute pancreatitis based on the 2012 revised Atlanta Classification. Gastroenterol Rep (Oxf) 6:127-131. doi: 10.1093/gastro/gox029.

13. Jain D, Bhaduri G, Jain P ( 2019) DIFFERENT SCORING SYSTEMS IN ACUTE ALCOHOLIC PANCREATITIS: WHICH ONE TO FOLLOW? AN ONGOING DILEMA. Arq Gastroenterol 56:280-285. doi: 10.1590/S0004-2803.201900000-53. 
14. Papachristou GI, Muddana V, Yadav D, O'Connell M, Sanders MK, Slivka A, Whitcomb DC (2010) Comparison of BISAP, Ranson's, APACHE-II, and CTSI scores in predicting organ failure, complications, and mortality in acute pancreatitis. Am J Gastroenterol 105:435-441; quiz 442. doi: 10.1038/ajg.2009.622.

15. Arif A, Jaleel F, Rashid K (2019) Accuracy of BISAP score in prediction of severe acute pancreatitis. Pak J Med Sci 35:1008-1012. doi: 10.12669/pjms.35.4.1286.

16. Vasudevan S, Goswami P, Sonika U, Thakur B, Sreenivas V, Saraya A (2018) Comparison of Various Scoring Systems and Biochemical Markers in Predicting the Outcome in Acute Pancreatitis.Pancreas 47:65-71. doi: 10.1097/MPA.0000000000000957.

17. Lankisch PG , Pflichthofer $D$, Assmus $C$, Lehnick $D$ (1998) Which is the critical balthazar score value for indicating organ failure in acute pancreatitis? Gastroenterology 114:A477.doi: 1016/S00165085(98)81930-6.

18. Cho JH, Kim TN, Chung HH, Kim KH (2015) Comparison of scoring systems in predicting the severity of acute pancreatitis.World J Gastroenterol 21:2387-2394. doi: 10.3748/wjg.v21.i8.2387.

19. Haddad JD (2017) Acute Pancreatitis.N Engl J Med 376:597. doi: 10.1056/NEJMc1616177.

20. Mounzer R, Langmead CJ, Wu BU, Evans AC, Bishehsari F, Muddana V, Singh VK, Slivka A, Whitcomb DC, Yadav D, Banks PA, Papachristou GI (2012) Comparison of existing clinical scoring systems to predict persistent organ failure in patients with acute pancreatitis. Gastroenterology 142:1476-1482; quiz e15-6. doi: 10.1053/j.gastro.2012.03.005.

21. Jones MJ, Neal CP, Ngu WS, Dennison AR, Garcea G (2017) Early warning score independently predicts adverse outcome and mortality in patients with acute pancreatitis. Langenbecks Arch Surg 402:811-819. doi: 10.1007/s00423-017-1581-x.

22. Juneja D,Gopal P B , Ravula M ( 2010) Scoring systems in acute pancreatitis: Which one to use in intensive care units? J Crit Care 25:358.e9-358.e15. doi: 10.1016/j.jcrc.2009.12.010.

23. Kuo DC , Rider A C , Estrada P , Kim D, Pillow MT (2015) Acute Pancreatitis: What "s the Score? J Emerg Med 48:762-770. doi:10.1016/j.jemermed.2015.02.018.

\section{Tables}

Table 1 Comparison of gender, age, history of pancreatitis, DP, PE, HR, RR, and SBP between the two groups 


\begin{tabular}{lllll}
\hline Parameters & SAP group(n=280) & Non-SAP group(n=744) & $\chi^{2} / t / U$ value & $P$ value \\
\hline male/female $\llbracket \mathrm{n} / \mathrm{n} \square$ & $200 / 80$ & $352 / 392$ & 6.128 & 0.017 \\
Age (year) & $49.23 \pm 14.44$ & $50.43 \pm 14.38$ & -0.421 & 0.675 \\
History of pancreatitis (yes/no) & $80 / 200$ & $152 / 592$ & 0.931 & 0.349 \\
ADP (yes/no) & $168 / 112$ & $208 / 536$ & 10.981 & 0.001 \\
PE (yes/no) & $184 / 96$ & $96 / 648$ & 33.653 & 0.000 \\
HR (beats/min $\square$ & $125(110,141)$ & $86(73,93)$ & -7.522 & 0.000 \\
RR (beats/min $\square$ & $28(23,37)$ & $20(20,21)$ & -7.735 & 0.000 \\
SBP (mmHg) & $123.57 \pm 21.58$ & $126.19 \pm 21.98$ & -0.604 & 0.547 \\
\hline
\end{tabular}

SAP: severe acute pancreatitis; SP, severe pancreatitis; ADP, acute diffuse peritonitis; PE, pleural effusion; HR, heart rate; RR, respiratory rate; SBP: systolic blood pressure

Table 2 Logistic regression analysis of risk factors for severe acute pancreatitis

\begin{tabular}{lllllll}
\hline Parameters & $\beta$ & $S E$ & Wals & $P$ & OR & OR95\%CI \\
\hline PE & 2.042 & 0.842 & 5.873 & 0.015 & 7.704 & $1.478 \llbracket 40.163$ \\
HB & 0.075 & 0.025 & 8.920 & 0.003 & 1.078 & $1.026 \square 1.133$ \\
RR & 0.434 & 0.165 & 6.947 & 0.008 & 1.543 & $1.118 \llbracket 2.131$ \\
\hline
\end{tabular}

Table 3 The Ability of PE, HR, RR in prediction of severe acute pancreatitis in patients with acute pancreatitis

\begin{tabular}{lllllll}
\hline meters & AUC $(95 \%$ CI $\square$ & Youden's index & Cut-off value (points) & Sensitivity (\%) & Specificity (\%) & OR value \\
\hline res $/ \mathrm{no} \square$ & $0.764(0.662,0.866)$ & 1.528 & 0.5 & $65.70 \%$ & $87.10 \%$ & 12.938 \\
beats $/ \min \square$ & $0.932(0.875,0.989)$ & 1.742 & 95.50 & $91.40 \%$ & $82.80 \%$ & 1.594 \\
beats $/ \min \square$ & $0.921(0.855,0.986)$ & 1.764 & 22.5 & $82.90 \%$ & $93.50 \%$ & 1.151 \\
\hline
\end{tabular}

Table 4 RISAP score designed based on cutoff values of three parameters

\begin{tabular}{lll}
\hline Parameters & 0 point & 1 point \\
\hline PE(yes/no $\square$ & no & yes \\
HR(beats/min $\square$ & $\square 96$ & $\geq 96$ \\
RR(beats/min $\square$ & $\square 23$ & $\geq 23$ \\
\hline
\end{tabular}


Table 5 Comparison of gender, age, RISAP, RANSON, and BISAP scores between SAP and non-SAP groups

\begin{tabular}{|c|c|c|c|c|c|c|c|}
\hline \multirow[t]{2}{*}{ Groups } & \multirow[t]{2}{*}{ Number } & \multicolumn{2}{|c|}{ Gender (n) } & \multirow[t]{2}{*}{ Age (year) } & \multirow[t]{2}{*}{ RISAP score } & \multirow[t]{2}{*}{ RANSON score } & \multirow[t]{2}{*}{ BISAP score } \\
\hline & & male & female & & & & \\
\hline SAP group & 304 & 204 & 100 & $48.49 \pm 15.49$ & $2(1,2)$ & $3(1,4)$ & $2(1,3)$ \\
\hline non-SAP group & 436 & 244 & 192 & $47.08 \pm 15.50$ & $0(0,1)$ & $2(1,3)$ & $0(0,1)$ \\
\hline$\chi^{2} / t / U$ value & & 2.347 & & 0.606 & -9.501 & -3.710 & -8.520 \\
\hline$P$ value & & 0.131 & & 0.545 & 0.000 & 0.000 & 0.000 \\
\hline
\end{tabular}

Table 6 The ability of RISAP, RANSON, BISAP scores in predicting severe acute pancreatitis

\begin{tabular}{llllllll}
\hline meters & AUC & 95\%CI & Youden's index & Cut-off value (points) & Sensitivity (\%) & Specificity (\%) & OR value \\
\hline P & 0.892 & $0.842,0.941$ & 1.760 & 1.5 & 79.70 & 96.30 & 3.221 \\
SON & 0.657 & $0.576,0.739$ & 1.227 & 2.5 & 53.90 & 68.80 & 2.104 \\
$\mathrm{P}$ & 0.853 & $0.796,0.909$ & 1.614 & 1.5 & 75.00 & 86.40 & 2.210 \\
\hline
\end{tabular}

\section{Figures}




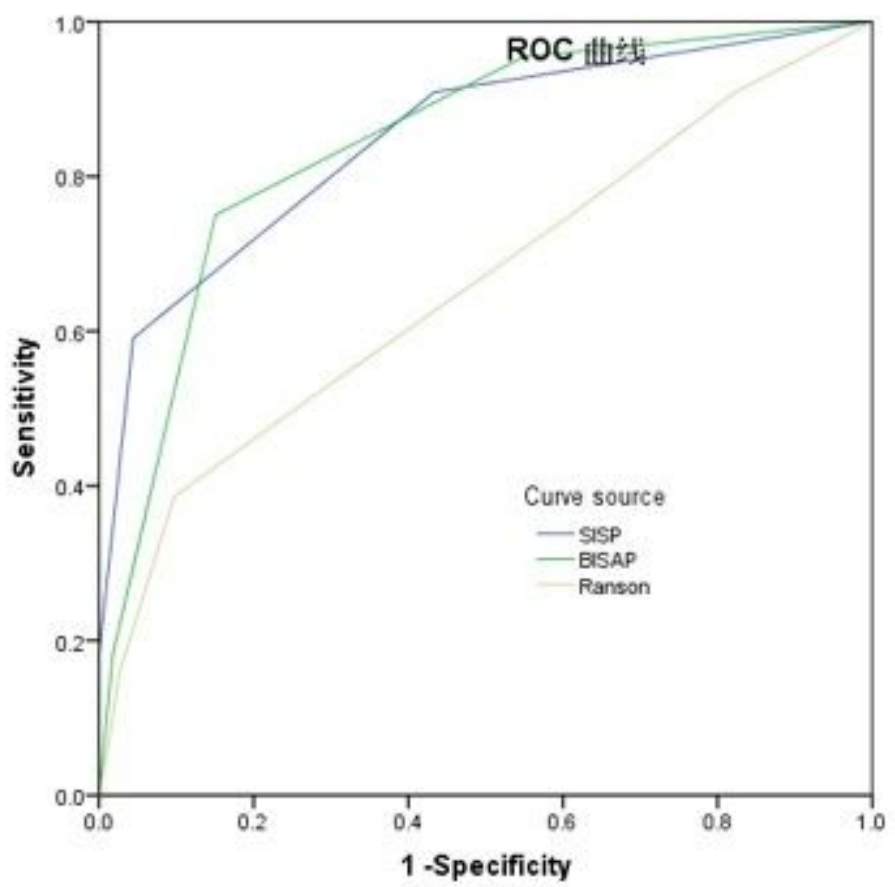

Figure 1

ROC curve for RISAP, RANSON, BISAP scores in predicting severe acute pancreatitis 